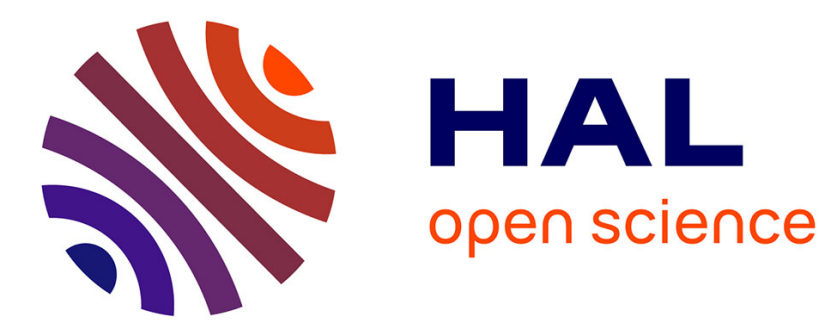

\title{
Non-Newtonian behaviour of flocculated suspensions P. Mills
}

\section{To cite this version:}

P. Mills. Non-Newtonian behaviour of flocculated suspensions. Journal de Physique Lettres, 1985, 46

(7), pp.301-309. 10.1051/jphyslet:01985004607030100 - jpa-00232515

\section{HAL Id: jpa-00232515 https://hal.science/jpa-00232515}

Submitted on 1 Jan 1985

HAL is a multi-disciplinary open access archive for the deposit and dissemination of scientific research documents, whether they are published or not. The documents may come from teaching and research institutions in France or abroad, or from public or private research centers.
L'archive ouverte pluridisciplinaire HAL, est destinée au dépôt et à la diffusion de documents scientifiques de niveau recherche, publiés ou non, émanant des établissements d'enseignement et de recherche français ou étrangers, des laboratoires publics ou privés. 
Classification

Physics Abstracts

$82.75 \mathrm{~K}-47.50$

\title{
Non-Newtonian behaviour of flocculated suspensions
}

\author{
P. Mills \\ Laboratoire de Biorhéologie et d'Hydrodynamique Physico-Chimique (*), \\ Université Paris VII, 2, Place Jussieu, 75251 Paris Cedex 05, France
}

(Reçu le 31 août 1984, révisé le 11 janvier 1985, accepté le 7 février 1985)

\begin{abstract}
Résumé. - Un modèle théorique est proposé pour décrire le comportement non-Newtonien des suspensions floculées. On suppose que le fluide immobilisé entre les particules formant les floculats est à l'origine de ce comportement. Une approximation de champ auto-consistant est utilisée pour estimer le rayon moyen des floculats s'écoulant dans un champ de cisaillement simple, et, par voie de conséquence, la quantité de fluide immobilisée dans les floculats. Une loi rhéologique est alors proposée où interviennent quatre paramètres physiques.

Abstract. - A theoretical model.is proposed to describe the non-Newtonian behaviour of flocculated suspensions. This behaviour is supposed to originate from the immobilized fluid between the particles which forms flocs. A self-consistent field approximation is used to estimate the mean radius of flowing flocs in a simple shear field, and, as a consequence, the amount of immobilized fluid in flocs. Then a rheological law is proposed in which four fixed physical parameters are involved.
\end{abstract}

\section{Introduction.}

The bulk rheology of rigid small spheres suspended in Newtonian fluids strongly depends on the nature of interactions between particles and on the particle size. Brownian motion and interparticle forces can equilibrate when the characteristic size of particles is below the micrometer, while hydrodynamic forces are predominant for macroscopic sizes.

For a large variety of colloidal suspensions (particle in diameter range 1 to $10 \mu \mathrm{m}$ ) Brownian motion is weak enough to be neglected, while interparticle interaction remains an important factor in the bulk rheology.

For a flocculated suspension, as a consequence of coupling between the microstructure of the suspension and the imposed flow, the characteristic size to be considered is the mean floc size instead of the individual particle diameter. As a matter of fact, at low shear rates, floc size may be considered as macroscopic. At high shear rates, even if flocs are reduced to individual particles, Brownian motion can be neglected when compared to hydrodynamic interactions.

Therefore, in the following, the non-Newtonian behaviour due to Brownian motion $[1,2]$ is not taken into account. On the contrary, our attention is focused on the coupling between microscopic physico-chemical properties and the rheological behaviour of colloidal suspensions.

(*) Laboratoire associé au CNRS, no 343. 
As remarked in [3] the study of flocculated suspensions at low shear rates is somewhat difficult because of the non-equilibrium nature of the structure at rest resulting from the weakness of Brownian motion.

At rest, when the particle volume fraction is above the percolation transition threshold, aggregates are supposed to form a three-dimensional network. When a finite stress is applied to the suspension, this structure breaks. Increasing the stress, aggregates are gradually disrupted and are finally reduced to individual (radius $a$ ). With decrease in stress, individual particles build up aggregates again. In a steady state, a dynamical equilibrium exists between the state of aggregation and the stress applied.

In section 2 we recall some experimental and theoretical results about the steady viscosity of a suspension of non-Brownian hard spheres as a function of the particle volume fraction $\phi$.

In section 3 we define the floc structure as a composite of randomly packed blobs characterized by a fractal dimension $D$. The non-Newtonian behaviour is supposed to be due to trapped fluid inside each floc. The volume of this trapped fluid is evaluated as a function of the mean particle coordination number $z$.

In section 4 a relation is proposed to describe the dynamical equilibrium between the mean radius of the fractal blobs and the shear stress applied.

In the last section, a rheological law is inferred by introducing an effective particle volume fraction in the rheological law related to a suspension of hard spheres. This effective volume fraction takes into account the trapped fluid inside the flowing flocs. These results are compared to experimental data related to flocculated suspensions. The most striking point of this model is that flocculated suspensions can be described by means of only four main physical parameters : the particle volume fraction, the energy related to pair interactions, the viscosity of the suspending fluid and the critical percolation concentration.

\section{Rheological law governing the rheology of a suspension of hard spheres.}

The flow of a dilute suspension of neutrally buoyant hard spheres exhibits a Newtonian behaviour due to the reversibility of the Stokes equation governing the flow around individual particle or doublets. Up to now, only the two first terms of the expansion of the bulk viscosity in terms of particle volume fraction have been rigorously inferred. The $\mathcal{O}(\phi)$ term was calculated by Einstein in 1906 [4], and the $\mathcal{O}\left(\phi^{2}\right)$ term was calculated much later by Batchelor [5]

$$
\frac{\mu}{\mu_{0}}=1+5 / 2 \phi+5.2 \phi^{2}+\mathcal{O}\left(\phi^{3}\right)
$$

The numerical coefficient of the $\mathcal{O}\left(\phi^{2}\right)$ term must be replaced by 5.2 when only the hydrodynamical contribution with a uniform distribution is considered. When the volume fraction $\phi$ is increased beyond the critical percolation concentration $\phi_{\mathrm{p}}$, the presence of an infinite transient cluster (see for instance [6]) prevents the use of equation (1) for the suspension viscosity.

It has been suggested in [7], as a conjecture, that the percolation transition must play a role in the variation of viscosity with concentration. As a consequence, in the following, we consider the suspension as concentrated if $\phi>\phi_{\mathrm{p}}$.

To calculate the apparent shear viscosity of a concentrated suspension of hard spheres in a Newtonian fluid we use a free cell model [8] which has been widely used for calculating the properties of concentrated suspensions. A cell consists of a particle surrounded by a shell of fluid and by the other particles. The apparent overall shear field is defined by :

$$
u_{y}, u_{z}=0 ; \quad u_{x}=\stackrel{\circ}{\gamma} y .
$$


The local shear rate $\stackrel{\circ}{\gamma}^{*}$ averaged over the fluid shell volume is :

$$
\stackrel{\circ}{\gamma}^{*}=\stackrel{\circ}{\gamma}\left(1-\frac{\phi}{\phi^{*}}\right)^{-1}
$$

where $\phi^{*}$ is the packing concentration of randomly packed spheres.

The dissipation per unit time and per unit volume expressed by means of the apparent shear viscosity and the apparent shear rate $\gamma$ must be identical to the dissipation per unit time in the fluid fraction (viscosity $\mu_{0}$ ) :

$$
\mu \dot{\gamma}^{2}=\mu_{0} \stackrel{\circ}{\gamma}^{* 2}(1-\phi)
$$

Hence, the apparent shear viscosity is :

$$
\frac{\mu}{\mu_{0}}=\frac{1-\phi}{\left(1-\phi / \phi^{*}\right)^{2}} .
$$

In the following, equation (2) is assumed throughout this paper to be the reference equation for a concentrated suspension of hard spheres with only hydrodynamic interactions [9].

To take particle interactions into account it has been suggested first in [10] and later in [11, 12] that an effective particle volume fraction be considered instead of the real volume fraction.

\section{Some conjectures concerning the structure of a concentrated flocculated suspension.}

Recently several theoretical models have been introduced to describe the growth of particle clusters by aggregation $[13,14,23]$. We consider here " the kinetic clustering of clusters " model generalized to allow for chemical effects. Recent numerical simulations performed for the space dimension $d=3$, reported in [23] lead to the choice $D=2$, where $D$ is the fractal (Hausdorff) dimension of clusters.

A characteristic length of every fractal cluster is the radius of gyration $R_{\mathrm{F}}$. This length is defined as follows :

$$
R_{\mathrm{F}}^{2}=\frac{1}{N} \sum_{i=1}^{N}\left(\mathbf{R}_{i}-\mathbf{R}\right)^{2}, \quad \mathbf{R}=\frac{1}{N} \sum_{i=1}^{N} \mathbf{R}_{i}
$$

where the summation covers all the $N$ particle sites of the cluster, and where $\mathbf{R}_{i}$ joins the origin and the centre of every $i$ particles. The gyration radius $R_{\mathrm{F}}$ is simply related to the Hausdorff dimension $D$ and to the number $N$ of particles per cluster :

$$
\left(\frac{R_{\mathrm{F}}}{a}\right)^{D} \sim N
$$

The particle density in a fractal structure is a decreasing function of the number $N$ of particles belonging to the structure when $D<3$. Therefore, in a concentrated suspension, fractal structure cannot grow indefinitely.

We will consider now, the suspension being at rest, the formation of a packed structure formed of fractal aggregate(s) able to fill the space between two planes, whatever the distance between planes. When the volume fraction $\phi$ is below the critical percolation concentration $\phi_{\mathrm{p}}$, this structure cannot appear [6]. Up to $\phi_{\mathrm{p}}, \phi$ is the volume fraction of isolated particles or of small aggregates. Above the percolation concentration $\phi_{\mathrm{p}}$, the suspension is formed of isolated particles or finite aggregates and of an infinite cluster able to connect every pair of points inside the suspension. To summarize, we can make the crude assumption that for $\phi>\phi_{\mathrm{p}}$, the partial volume fraction of isolated particles and small aggregates is $\phi_{\mathrm{p}}$ and that the partial volume fraction of 
the infinite structure is $\phi-\phi_{\mathrm{p}}$. This infinite structure is supposed to be constructed with fractal blobs (volume fraction $\phi_{\mathrm{F}}$ ), the characteristic dimension of which is $\hat{R}_{\mathrm{F}}$ at rest.

These blobs are packed with the volume fraction $\phi^{*}$. Therefore :

and :

$$
\phi-\phi_{\mathrm{p}}=\phi^{*} \phi_{\mathrm{F}}
$$

$$
k_{0}\left(\phi-\phi_{\mathrm{p}}\right)=\frac{N a^{3}}{\hat{R}_{\mathrm{F}}^{3}}, \quad k_{0}=\frac{1}{\phi^{*}} .
$$

When $\phi$ and $\phi_{\mathrm{p}}$ are equal, the fractal characteristic length $\hat{R}_{\mathrm{F}}$ becomes infinite and the volume fraction $\phi_{\mathrm{F}}$ tends to zero.

When $\phi>\phi_{\mathrm{p}}$, the structure of the infinite cluster is no longer purely fractal but formed of fractal blobs packed with the volume fraction $\phi^{*}$. Let $D$ be the fractal dimension of blobs :

$$
\frac{\hat{R}_{\mathrm{F}}}{a}=\left[k_{0}\left(\phi-\phi_{\mathrm{p}}\right)\right]^{1 /(D-3)}, \quad D \neq 3, \phi>\phi_{\mathrm{p}} .
$$

We pointed out in the preceding section that in order to take particle interactions into account, it has been suggested that equation (2) can be written :

$$
\frac{\mu}{\mu_{0}}=(1-\phi) /\left(1-\phi_{\text {eff }}\right)^{2}, \quad \phi_{\text {eff }}=k \phi
$$

where $\phi_{\text {eff }}$ is the effective particle volume fraction equal to the sum of the real volume of particles plus the trapped fluid between particles in contact. When interactions are purely hydrodynamic, the $k$ factor reduces to the « hydrodynamic factor $" k_{0}$ : it is the ratio of the effective solid volume to a geometrical volume fraction

$$
\begin{aligned}
& \phi_{\text {eff }} \rightarrow k_{0} \phi, \quad k_{0}=\frac{1}{\phi^{*}} \\
& \phi_{\text {eff }}=k^{\prime} k_{0} \phi .
\end{aligned}
$$

We can make the assumption that a certain amount of liquid around the "contact point " of two spheres and the spheres have no relative velocity. We must emphasize here that in our model the trapped fluid does not include the trapped liquid which cannot be liberated by shearing. As a consequence of this statement the elementary particle of our model may be an aggregate of several solid spheres which are strongly coagulated. Another consequence in practice is that an amount of trapped liquid which is not liberated by shearing in ordinary viscometers can be liberated by applying a powerful sonification to the suspension.

3. 1 Mean VAlue of THE COORDinAtion NUMBER. - Let $z$ be the mean number of contacts for every sphere belonging to a floc and let $\beta a^{3}$ be the trapped fluid per contact. Therefore, the $k^{\prime}$ factor can be expressed as a function of the mean coordination number $z$ :

$$
k^{\prime}=\frac{N a^{3}+N z \beta a^{3}}{N a^{3}}=1+\beta z .
$$

Let $z_{0}$ be the mean value of the coordination parameter in an infinite fractal floc. The coordination number must be lower than $z_{0}$ in a fractal floc whose radius $R_{\mathrm{F}}$ is finite since the particles at the floc surface must have fewer than $z_{0}$ contacts with their neighbours. On the other hand, the constancy of $z_{0}$ throughout the fractal structure can be inferred by some arguments analogous to those used in polymer studies [15]. A pair correlation function may be defined as follows : we choose one particle at random in the fractal structure. Then we determine the number density 
of the other particles at a distance $r$ of the first and we average the result over all possible choice of the first particle. This function scales like the density inside the fractal floc :

$$
g(r) \sim \frac{1}{R^{3-D}}
$$

Then, $z=z_{0}$ everywhere except on the surface. Here and in the following, surfaces are not defined as fractal surfaces but as classical surfaces corresponding to spherical volume of blobs, with radius $R_{\mathrm{F}}$.

On the floc surface the coordination number is the fraction $\alpha z_{0}$ of the coordination number inside the floc. Hence :

$$
z=z_{0}-(1-\alpha) z_{0} \frac{\Delta N}{N}
$$

where $N$ is the number of particles in a fractal blob and $\Delta N$ is the number of particles at the surface

Hence :

$$
\frac{\Delta N}{N}=D \frac{\Delta R_{\mathrm{F}}}{R_{\mathrm{F}}}, \Delta R_{\mathrm{F}} \sim a .
$$

$$
z=z_{0}\left[1-(1-\alpha) D \frac{a}{R_{\mathrm{F}}}\right]
$$

when the blob radius $R_{\mathrm{F}}$ tends to the particle radius $a$ (that means that there is no permanent contact between particles) the coordination number must tend to zero

$$
z=z_{0}\left(1-\frac{a}{R_{\mathrm{F}}}\right)
$$

Finally, considering equations (5) and (6) :

$$
k^{\prime}=1+\beta z_{0}\left(1-\frac{a}{R_{\mathrm{F}}}\right) .
$$

Now using equation (4) we can point out that the suspension viscosity becomes infinite when the effective volume $\phi_{\text {eff }}$ tends to unity. We state that this occurs when the gyration radius $R_{F}$ reaches a maximum. Recalling equations (3) and (7) :

$$
\begin{gathered}
\frac{a}{\hat{R}_{\mathrm{F}}}=\left[k_{0}\left(\phi-\phi_{\mathrm{p}}\right)\right]^{1 /(3-D)} \\
\phi_{\text {eff }}=k \phi=k_{0} \phi\left[1+\beta z_{0}\left(1-\frac{a}{\hat{R}_{\mathrm{F}}}\right)\right]=1 .
\end{gathered}
$$

From (3) and (8) we can deduce the $z_{0}$ value when the Hausdorff dimension is $D \simeq 2$ :

$$
\beta z_{0}=\frac{1}{k_{0} \phi} \frac{1-k_{0} \phi}{1-k_{0}\left(\phi-\phi_{\mathrm{p}}\right)}
$$

$z_{0}$ being the highest value of the fractal coordination number $z$.

From (7) and (9) :

$$
\phi_{\text {eff }}=k_{0} \phi\left[1+\frac{1}{k_{0} \phi} \frac{1-k_{0} \phi}{1-k_{0}\left(\phi-\phi_{\mathrm{p}}\right)}\left(1-\frac{a}{R_{\mathrm{F}}}\right)\right] .
$$




\section{Equilibrium size of flocs in a simple shear flow.}

Numerous theoretical and experimental studies have demonstrated that a shear flow enhances the aggregation process in dilute suspensions until the flocs which are formed in the flow are large enough to be destroyed by the shear stress which is acting on their surface. Monte Carlo studies of the coalescence and breakage of aggregates show the effect of shear rate on the aggregate size distribution [16]. It has been observed that, as the shear rate is increased, the relative frequency of the smaller aggregates increases and that of the larger aggregates decreases correspondingly. These results allow us to make the assumption that the main features of this process are still applicable for concentrated suspensions. Flocs forming the following bulk suspension can enlarge until they reach a critical size corresponding to a dynamical equilibrium at a given shear stress. When a floc appears in the flow with a size above this critical size, it is immediately disrupted. Therefore, at a given shear stress $\tau$, the floc radius must satisfy an equilibrium equation :

$$
R_{\mathrm{F}}=F(\tau) \text {. }
$$

We will now consider an elastic floc as in [17]. The energy required to rupture a floc consists of two parts : $E_{\mathrm{R}}$, the energy required to rupture the links which have been formed between the particles inside the floc, and $E_{\mathrm{D}}$, the energy needed to stretch (but not break) the elastic links between particles within the floc as the tension is transmitted from the shear field to the doublet contact area.

We must emphasize at this point that the energy required to stretch the links between particles is proportional to the number of links per unit volume. But the number of links in the rupture area is proportional to the number of links per unit surface. Then, the main contribution to the energy dissipation may come from stretching bonds within the floc rather than breaking those between two parts of the floc.

We demonstrated in [18] a relationship between the critical deformation of an elastic sphere just before rupture, the surface energy $\Gamma$ and the sphere radius $R$ :

$$
\tau_{\mathrm{D}} \sim \frac{\Gamma}{R}
$$

To describe the rupture process between two surfaces whose areas are proportional to $R^{2}$, the Deryaguin equation [19] can be used :

$$
F_{\mathrm{R}}=2 \pi R \Gamma
$$

where $F_{\mathrm{R}}$ is the rupture force and $\Gamma$ a surface energy. If :

then :

$$
F_{\mathrm{R}} \sim R^{2} \tau_{\mathrm{R}}
$$

and :

$$
R \tau_{\mathrm{R}} \sim \Gamma
$$

$$
R\left(\tau-\tau_{\mathrm{D}}\right)=\Gamma
$$

Hence $\tau$ and $\tau_{\mathbf{D}}$ are proportional :

$$
K \tau=\tau_{\mathbf{D}}
$$

The surface energy $\Gamma$ in the section of rupture $S_{\mathrm{R}}$ is defined as follows. Let $N_{\mathrm{c}}$ be the number of particles in $S_{\mathrm{R}}$ :

$$
N_{\mathrm{c}}=\frac{N}{R^{3}} R^{2} a=\frac{N a}{R}
$$

where $N$ is the total number of particles in the floc (radius $R$ ). 
The surface energy is :

$$
\Gamma=\frac{N_{\mathrm{c}} \gamma a^{2}}{R^{2}}=\frac{N \gamma a^{3}}{R^{3}}
$$

where $\gamma a^{2}$ is the bond energy corresponding to every pair interaction between elementary particles. We deliberately ignore here the exact nature of the physico-chemical interactions generating $\gamma$ (Van der Waals, electrostatic, polymer bridging, etc.). Finally, taking into account the fact that Deryaguin's equation leads to :

$$
\frac{R}{a}=\left[\frac{A \gamma}{a \tau}\right]^{1 /(4-D)}
$$

and that the floc radius cannot decrease to zero but tends to the limit $R=a$ when the shear stress $\tau$ becomes infinite, we propose as the equilibrium equation :

$$
\frac{R-a}{a}=\left[\frac{A \gamma}{a \tau}\right]^{1 /(4-D)}
$$

where $A=(1-K)^{-1}$.

To define the shear stress we treat the multifloc interactions via the self-consistent field approximation. This involves surrounding a spherical unit floc, consisting of interacting rigid particles, with a continuum having the mechanical properties of the bulk suspension. Introducing in equation (11) the Hausdorff dimension $D \sim 2$ :

$$
\frac{a}{R}=\frac{1}{1+\left(A \tau^{*} / \tau\right)^{1 / 2}}
$$

where we put $\frac{\gamma}{a}=\tau^{*}$.

In an experimental study of flow of dilute coagulated sols [20] the authors found that the average slope of the $\log$ (shear rate) $-\log$ (radius) curve is $\sim 0.42$ and does not appear to depend in any way on the bond energy.

With a fractal dimension $D=2$, equation (11) would give the value 0.5 for the above mentioned slope. This slight discrepancy can be explained by a possible crossover from the chemically limited to the diffusion limited model.

\section{Viscosity of a flocculated suspension as a function of the imposed shear rate.}

At this stage of our analysis, we are able to express the effective volume fraction as a function of the shear stress inside the suspension

$$
k=1+\beta z_{0}\left(1-\frac{a}{R}\right)
$$

According to equations (10) and (11) we can write :

$$
\phi_{\text {eff }}=k^{\prime} k_{0} \phi=k_{0} \phi\left[1+\frac{1}{k_{0} \phi} \frac{1-k_{0} \phi}{1-k_{0}\left(\phi-\phi_{\mathrm{p}}\right)}\left[\frac{\left(\frac{A \tau^{*}}{\tau}\right)^{1 / 2}}{1+\left(\frac{A \tau^{*}}{\tau}\right)^{1 / 2}}\right]\right]
$$


Introducing the value of the effective volume fraction given by equation (12) in the viscosity equation (4) we obtain after some simple algebra :

$$
\tau^{1 / 2}-\left(A \tau^{*}\right)^{1 / 2} \frac{k_{0}\left(\phi-\phi_{\mathrm{p}}\right)}{1-k_{0}\left(\phi-\phi_{\mathrm{p}}\right)}-\frac{(1-\phi)^{1 / 2}}{1-k_{0} \phi} \mu_{0}^{1 / 2} \stackrel{\circ}{\gamma}^{1 / 2}\left[1+\left(\frac{A \tau^{*}}{\tau}\right)^{1 / 2}\right]=0
$$

Focusing our attention on the rheological properties of the suspension at low shear rates, we can calculate the value of the yield shear stress when the shear rate $\dot{\gamma}$ tends to zero

$$
\tau_{0}=A \frac{k_{0}^{2}\left(\phi-\phi_{\mathrm{p}}\right)^{2}}{\left[1-k_{0}\left(\phi-\phi_{\mathrm{p}}\right)\right]^{2}}\left(\frac{\gamma}{a}\right)
$$

An alternative manner to write equation (14a) is to express the energy $\varepsilon$ per bond :

$$
\tau_{0}=A \frac{k_{0}^{2}\left(\phi-\phi_{\mathrm{p}}\right)^{2}}{\left[1-k_{0}\left(\phi-\phi_{\mathrm{p}}\right)\right]^{2}} \frac{\varepsilon}{a^{3}} .
$$

The shear stress dependence on the volume fraction $\phi$, on the energy per bond $\varepsilon$, and on the particle radius $a$, which was experimentally determined in [21] for coagulated sols, confirms the validity of equation (14b).

A simplified expression for equation (13) can be obtained in the limit range $\tau \gg \tau^{*}$ :

$$
\tau^{1 / 2}=\tau_{0}^{1 / 2}+\mu_{\infty}^{1 / 2} \stackrel{\circ}{\gamma}^{1 / 2}
$$

where $\mu_{\infty}=\frac{\mu_{0}(1-\phi)}{\left(1-k_{0} \phi\right)^{2}}$.

Equation (15) is exactly Casson's equation [22]. When $\phi \gg \phi_{\mathrm{p}}$ the ratio of the apparent viscosity $\mu$ to $\mu_{\infty}$ can be expressed as a function of the dimensionless variable $\stackrel{\circ}{\Gamma}=\stackrel{\circ}{\gamma} / \circ_{0}$, where $\stackrel{\circ}{\gamma}_{0}$ is related to the yield shear stress $\tau_{0}$ by :

$$
\begin{gathered}
\tau_{0}=\mu_{\infty} \stackrel{\circ}{\gamma}_{0} \\
\frac{\mu}{\mu_{\infty}}=\frac{1}{4}\left[\stackrel{\circ}{\Gamma^{-1 / 2}}+1+\sqrt{\left(\stackrel{\circ}{\Gamma}^{-1 / 2}+1\right)^{2}+4\left(\frac{\mu_{0}}{\mu_{\infty}}\right)^{1 / 2} \frac{1}{k_{0} \phi} \stackrel{\circ}{\Gamma}^{-1 / 2}}\right]^{2} .
\end{gathered}
$$

When viscometric studies are performed in the low $\stackrel{\circ}{\Gamma}$ range (and correspondingly for high bond energy values) the relationship between apparent viscosity and shear rate can be represented by a power law.

\section{Conclusion.}

This model proposes a rheological state equation where, for a given fractal structure, four physical parameters are involved. We consider that this analysis constitutes progress in contrast with numerous non-Newtonian viscosity equations which use several phenomenological parameters. Despite the frequent use of scaling concepts in its elaboration, this rheological law reflects quite well the behaviour of sheared flocculated suspensions. 


\section{References}

[1] Krieger, I. M., Adv. Colloid Interface Sci. 3 (1972) 111.

[2] Mills, P., Snabre, P., Quemada, D., Revue Phys. Appl. 16 (1981) 523.

[3] Russel, W. B., J. Rheol. 24 (3) (1980) 287.

[4] Einstein, A., Ann. Phys. Leipzig 19 (1906) 289 and ibid. 34 (1911) 591.

[5] BATChelor, G. K., J. Fluid. Mech. 83 (1977) 97.

[6] Broadbent, S. and Hammersley, J., Proc. Camb. Philos. Soc. 53 (1957) 629.

[7] De Gennes, P. G., J. Physique 40 (1979) 783.

[8] HAPPEL, Jr., AIchE J. 4 (1958) 197.

[9] We remark that the shear viscosity expanded in terms of the volume fraction $\phi$ is (when $\left.\phi^{*}=0.58\right)$ : $\frac{\mu}{\mu_{0}}=1+2.45 \phi+5.47 \phi^{2}+11.58 \phi^{3}+\mathcal{O}\left(\phi^{4}\right)$. The $\mathcal{O}(\phi)$ and $\mathcal{O}\left(\phi^{2}\right)$ terms are very close to those calculated by a more rigorous approach.

[10] Vand, V., J. Phys. Colloid. Chem. 62 (1948) 277.

[11] GillePsie, T., in Colloid and Interface Science, Vol. 4, M. Kerker Ed., 1976.

[12] Quemada, D., Rheol. Acta 17 (1978) 632.

[13] Muthuxumar, M., Phys. Rev. Lett. 50 (1983) 839.

[14] Jullien, R., Kolb, M. and Botet, R., J. Physique Lett. 45 (1984) L-211.

[15] De Gennes, P. G., Scaling concepts in polymer Physics (Cornell University Press) 1979.

[16] Gupta, S., Saraf, D., Pandey, B., Colloid Polymer Sci. 251 (1979) 663.

[17] Firth, B., Hunter, R., J. Colloid Interface Sci. 57 (2) (1976) 266.

[18] Bitbol, M., Mills, P., J. Physique Lett. 45 (1984) L-775.

[19] Deryaguin, B., Muller, V. and Toporov, Y., J. Colloid Interface Sci. 53 (1975) 314.

[20] Hunter, R., Frayne, J., J. Colloid Interface Sci. 76 (1) (1980) 107.

[21] FIRTH, B., J. Colloid Interface Sci. 57 (2) (1976) 257.

[22] Casson, in : Rheology of disperse systems, C. C. Mills, Ed. (Pergamon Press, London) 1959, p. 84.

[23] Kolb, M. and Jullien, R., J. Physique Lett. 45 (1984) L-977. 